\title{
Humanizing Architecture in a Materialistic World Using Symbols and Morals
}

\author{
Aly Mohamed El Husseiny ${ }^{1}$, Ahmed Aly El Husseiny² \\ ${ }^{1}$ Architecture Department, Faculty of Engineering, University of Minia, Minia 61519, Egypt \\ ${ }^{2}$ Architecture Department, Faculty of Engineering, Cairo University, Cairo 12613, Egypt \\ alhusseiny14@yahoo.com
}

\begin{abstract}
Architectural schools of design overwhelmingly adapt to cultural backgrounds of societies they target. This paper distinguishes between ideologies that generated Western, contemporary architecture, and on the other hand, the values of traditional Arab communities. The paper aims at parrying architectural plastic formations that are irrelevant to the local Arab discourse. The paper rediscovers a value oriented architecture that is capable of moving spiritual feelings towards the built environment, even if its formalistic and visual attractiveness is controversial. The paper demonstrates examples of what can be called "sincere" architecture rather than stunning and sight-startling products that apparently or superficially hold value.
\end{abstract}

Keywords: Human architecture; spiritual architecture; symbolism; social building; experiencing architecture

eISSN 2398-4295 @ 2018. The Authors. Published for AMER ABRA cE-Bs by e-International Publishing House, Ltd., UK. This is an open-access article under the CC BY-NC-ND license (http://creativecommons.org/licenses/bync-nd/4.0/). Peer-review under responsibility of AMER (Association of Malaysian Environment-Behaviour Researchers), ABRA (Association of Behavioural Researchers on Asians) and cE-Bs (Centre for EnvironmentBehaviour Studies), Faculty of Architecture, Planning \& Surveying, Universiti Teknologi MARA, Malaysia.

DOI: http://dx.doi.org/10.21834/ajbes.v3i9.66 


\subsection{Introduction}

Buildings can be seen as mirrors that reliably reflect the identity of the societies that contain and incubates them. Even the architects who trigger them have no power to apply trends aside the desires of a society or its representatives. The modernity and materialistic progression that buildings reflect in the modern Western World are not unusual for advanced, industrial, and developed nations. On another hand, what seems odd is that local Arab countries, specifically with different cultures and norms, have been adopting similar languages and reflections along the past few decades. While both thought and product are distant from Arab culture, the mimicry of local communities that inclined towards formalistic architecture driven by materialistic values and market influences seems to be disoriented. Local Arab architects drifted with the stream of Modernism and even with the "semblances" of postmodern movements especially in the contagious state of globalization.

The paper rapidly reviews epistemic references of modern architecture and argues the conformance of its basics with local traditions. Subsequently, the paper demonstrates contemporary attempts of the author to apply designs that translate the theoretical background into practical application, hopefully, conducting socially and psychologically determined "human" environments.

The paper does not aim at the assessment of different projects in an attempt to evaluate their success or failure. If it had aimed at doing so, empirical research would have appeared to be the appropriate method. Instead, the paper is to show the distinctions between two approaches of design that have emanated from two different visions or perspectives, that of the Western discourse and our local traditional collective consciousness. In doing so, the paper shows strong dependency on a methodology of the "participant observer", in this case, the designer and user. To extract the values of local behavioral mores, the research had to strive to a more subjective and phenomenological methodology. This methodology requires the researchers' direct experience and deep contact with the projects under study.

\subsection{Literature Review}

\subsection{Mind Bias to materialistic forms}

The two illustrated cases in fig.1 present two states of the human body that are quite different. The first at the right (b), shows a position, freezed in a fraction of a second, to show how a human body can perform astounding movements. This position is a result of long concentration and hard training not only by the gymnast herself, but also it is a result of an effort of a big team of participants.

Human body in the case (b) draws attention to cleverness that cannot be usually performed by an ordinary person in daily life. On the other hand, the case (a) presents an ordinary state of the body that may not attract any attention. A comparison between the two states may help clearly point to the paper's proposition. While the astonishing case of somersault on a balance beam may be an attractive position that a human can hardly achieve, the other state of the body is no less miraculous for an attentive observer. For a 
supposed outsider, shape of a human body balance may be much more logical to be normally settled horizontally on four legs like many animals, than erecting vertically on just two feet. Human long proportioned body would rather be laid on the ground like many reptiles than stand in a contradictory state to balance principles of masses. A pencil in an analogous shape of body cannot be stable when left standing on its extremity point. However, the human body can walk, run, bend, carry weights with one hand, and even stand on a single foot, to amaze any truthful arbitrator observer. The remarkable non logic kinetic situations have never drawn attention due to one's taking for granted the easiness of their happening.
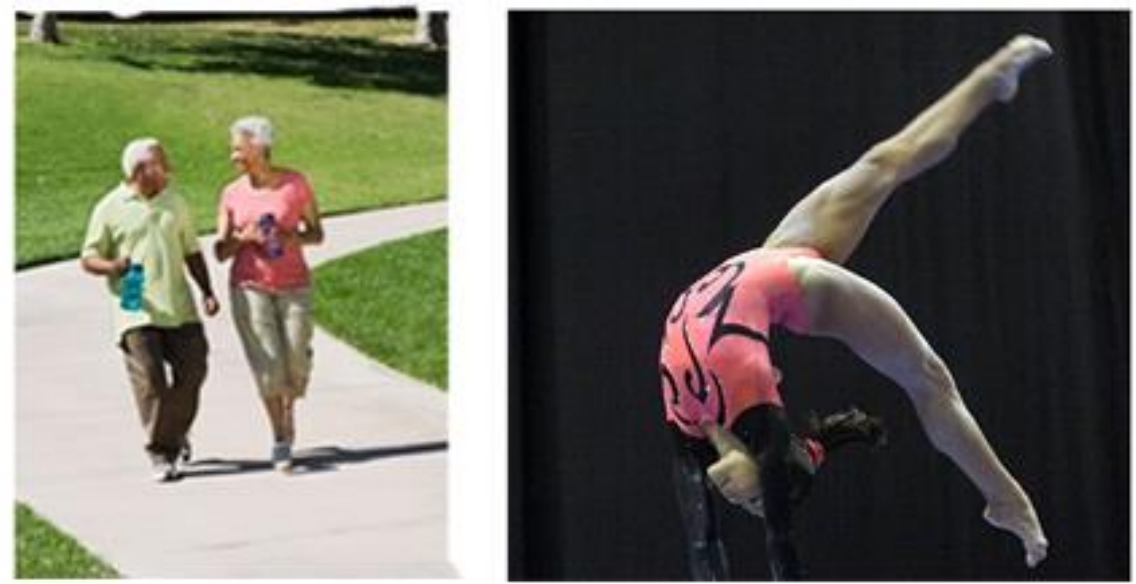

Fig.1. (a) Familiarity of normal satisfaction; versus provisional impressive pretention

(b); source: Gymnastics Inside magazine.(2012)

http://perfect10.rustyparts.com/wp-content/uploads/hong_1.jpg

The presented two states of the body are so vital to get along an assessment. The gymnastic made up movement had a cost that is relatively high in a sense of time, effort or even as subordinate to a sophisticated scientific knowledge of anatomy. According to the cost, what were the benefits? The gymnastic glimpsing movement drew anyone's attention to a cleverness embedding a message of superiority opposing incapacitation of others. The dangerous calculated state of the body in a totally unstable position might be conducted to be appreciated by applauses of spectators. On a scale of daily life, it may have no direct benefit or use other than splurge of an achievement that does not last for even a minute. Probably the performance presented itself as a goal to achieve. The difficulty act may deliver a message to other players of superiority. That is what architects had mainly expressed when they transformed the relaxed mode of habitual, peaceful way of living into a startling state within a contest where the winner may be acknowledged as a champ.

The paper seeks relating architecture to humanistic appreciation that raises its value. In the traditions of Muslim's sophism, anything always has two faces, one is its material structure, and the other is the embedded meaning. When a thing becomes old it tends to be broken and unused, then it ends by being worthless. On the contrary, meaning lasts even 
when its material container becomes old, or even disappears, thanks to the storage of memory in a human mind. A smile then, although its material component is too light, it may mean a powerful feeling that may last for time life. In other domains, artists lifted up their products' value when they understood the emotional, human effect in their works, especially in the cinema drama, songs, literature, and painting, while most architects were busy with high-end technology and using dazzling materials. In that sense, symbolism seemed to be an appropriate launching point to conduct to meaningful architectural compositions.

\subsection{Symbolism in Western buildings.}

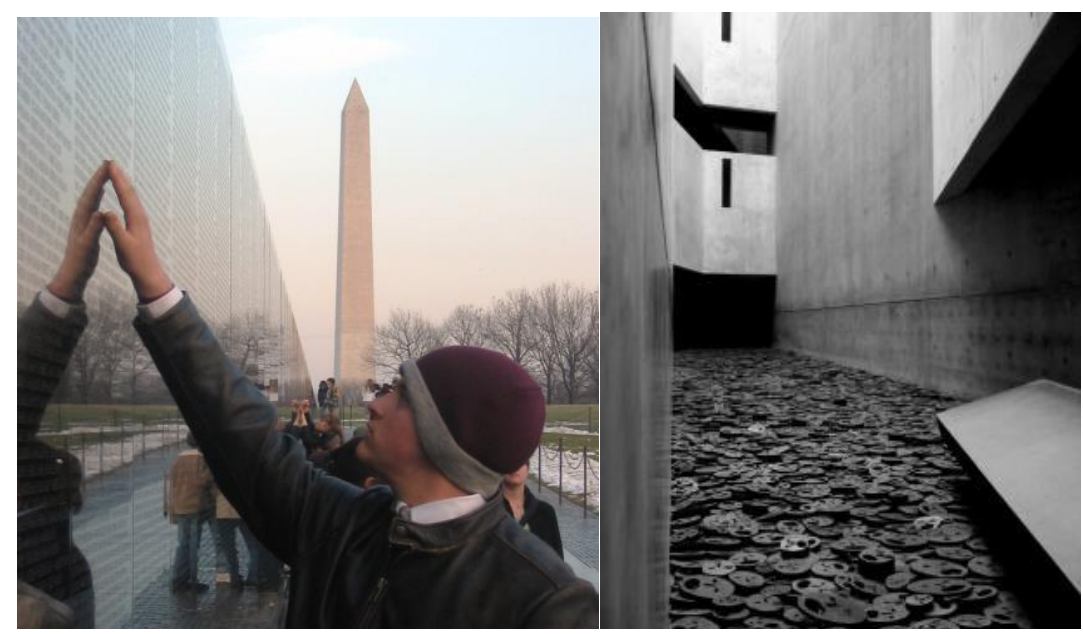

Fig.2. (a): Touching the Memorial Wall; source: Wikimedia, (2005).

http://en.wikipedia.org/wiki/File:TouchWall.jpg. And (b) the "Memory Void" where a visitor is invited to walk on metallic faces in the Jewish Museum; source:

http://www.katrinkalden.net

A few number of the western architectures succeeded to represent valuable symbolic values in the sense of the paper's aim. The Memorial Wall of Washington DC is one of the successful projects because its highly appreciated implicit symbolism. It has taken tenth ranking on the list of America's favorite Architecture by the American Institute of Architects. The memorial consists of two gabbros' walls that retain the earth ground behind them. Engraved names of martyrs of the Vietnam War appear on highly reflective black stones. Visitors can see their reflections on the stone wall simultaneously with the engraved names. This aims at symbolically connecting the past and present. The symbolic theme quietly presented in a park mixes simplicity and clarity. The embedded passion of sacrificing oneself for others is full of meaning. This meaning presents itself in easy architectural vocabulary that quietly expresses sorrow that does not overlook values of pride. 
The Jewish Museum of Berlin is another project that obviously reflected a symbolic value. The building's form resembles a warped Star of David. An irregular matrix of windows spreads in all orientations across the building's facade. 10000 steel punched faces scattered over the ground of the "Memory void", the only accessible voided space in the Libeskind building. Circulation guides the visitors to walk through the void, producing distressing sounds by stepping over the metal faces. While the whole design succeeded to lead sympathy towards Jews' history in an atmosphere of gloom, the experience of walking on faces may lead the visitor to be partially blamed to be a part of torturing and killing children. The building has too much sophistication that orients the visitor towards a single idea that the architect probably made sure the visitor has taken. .

\subsection{Design attempts to insert humanistic values.}

The question to be imposed now is: does only monumental symbolic form lead to humanism?. The paper presents a personal experience to practice humanistic design through two examples of the author's design of functional public buildings. In the first one, the designer adopted a similar line of experiencing symbolism in an everyday functional building. The other is a different approach where social interaction is the motive of humanism spirit.

The first attempt is a design presented for a primary school design competition. It is to be located in rural regions in order to fix a socio-cultural image of a sense of inferiority attached to the Egyptian peasant. The project's suitability for the target increases its opportunity since it targets children's minds in the first place. The design manifests the findings of a former study of the author investigating children's image of school buildings ( $E$ I Husseiny, 1996). The paper gave attention towards some architecture items that form the

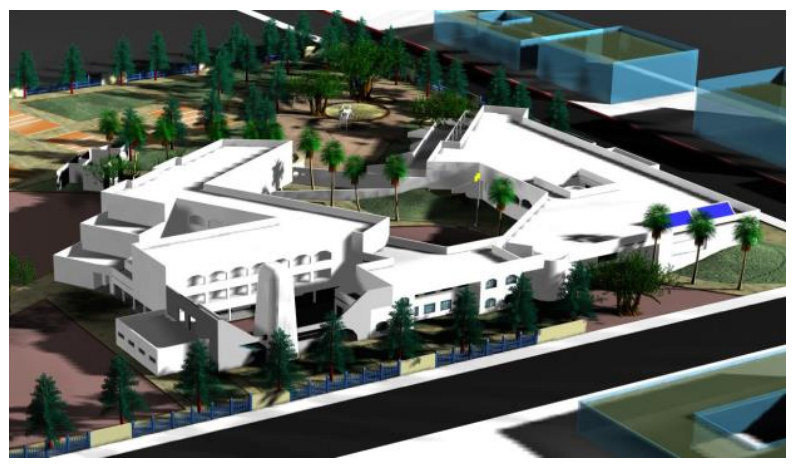

Fig.4. liberated masses of the primary school prototype in rural regions showing the entrance façade and main features of the building; source: the authors, (2001) 
school image to children: the gate, fences, the recreation court, the flag, hobbies, and the teacher are what students noticeably consider and express in their schools' images. The school masses transgressed the usual rigid rectangular form to decrease formality senses that do not match the actual rural environment. The main entrance facade was green earth sheltered in a hill like feature to give a message that planting is the source of beauty of this school, and earth in its honourable value to farmers constitutes a part of the entrance wall. A single Banyan tree (Ficus bengalensis) is to be planted to give intimacy in front of the main entrance. At the same time, it plays the role of a land mark that may last for decades and will carry generations' childhood memories to be attached with the school (fig.4).

Since the fence counts as the actual school facade to a child, emphasis of his attachment to the place manifests by displaying the highly appreciated children's agriculture hobby along the main elevation. A competitive agricultural activity may help a convergence of sons and fathers, discussing technicalities to enhance plants productivity and may lead to exchange pride ongoing the subject of agriculture. It may partially help to withdraw what seems to be evident in rural Egypt: a common hope of parents to see their children as doctors and engineers rather than practicing fathers' disgraceful career of planting. Extending the green area to the internal court through the entrance gate is a continuity relationship between the school and the village community; it then ends up with an internal green hill that may help children to enjoy playing. Up the hill, children may already reach half way to go to the first floor. Instead of using the traditional staircase that is still useable by teachers, children can continue to climb rope ladders on the adjoining wall to reach the hobby rooms. Pigeonhole is a useful internal feature that can be seen from outside and provides an animate excitement by spreading pigeons in the school's open spaces. A sudden takeoff fly of birds' masses to leave the ground when children scramble to the courtyard at the recreation time, or a visiting white pigeon on a window base of the class may present captivating daily scenes. A familiar interaction with living birds integrates the village productive aviculture. Design of the buildings' facades appears in pure mass forms dismissing non valuable details to match a minimalism value to appreciate.

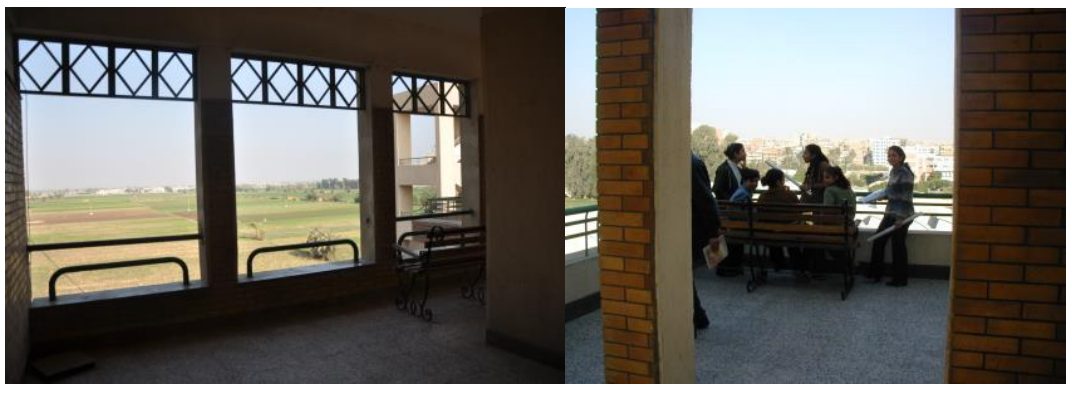

Fig.5. (a) Corridors of the building are framing landscape panoramas, and (b) terraces are designed as static spaces for large numbers of students to gather; source: the authors, (2011). 
The second building is an extension building of the faculty of Engineering at Minia Universtiy in Egypt. The building design presents it as a social building, to appreciate the social life of students in this livable youth period. An emphasis on creating interactive general spaces with human behaviour is eminent. The design provides static spaces as main components of the plan structure. Even corridors are wide enough to decrease their dynamism in order to keep students staying in the building's spaces. Partial static spaces with different sizes, arranged throughout the building, invite different group sizes to communicate. Increasing a visual contact between different floors through different open internal courts and terraces on the outside facades increased communication. The building is adjacent to cultivated lands; it directs the view to the remarkable landscape through openings along corridors and spaces. These stimuli are tools that add pleasure and help remain in the building. The corridors are windowless to increase a direct visual exposure to nature without any physical or symbolic obstruction, especially when preventing a possible impact of glass cleaning lack often found in public buildings. As the building functions for more than ten years now, it demonstrates a social appeal to human ties between students themselves and reinforced ties between the faculty staff and students, as well.

\subsection{Discussion}

Beauty is particularly relevant to goodness, compassion, sympathy. Human architecture is that architecture that relates buildings to transcendent qualities. Physical beauty or formalism, in fact, does not last for long since its perception is mostly superficial especially when routinely observed. Symbolism is one vital way to lend humanism to a building but is not exclusive.

\subsection{Types of symbolism}

Architectural symbolism may provide meanings to a building, but not all building types are suitable to different types of symbolism. We may summarize in the following types:

Artistic symbolism may be useful in monumental iconic buildings to be visited once in a lifetime or at long intervals of time. It represents just a message to be read, but the building does not have actual use rather than being a piece of art. It bears a lot of artificiality in order to highlight an idea.

Embedded symbolism through normal use of a functional building is much more moderately hidden to respect the user's comprehension and cultural capabilities. Although getting used to certain values may gradually weaken interest, meaning will still be retrieved now and then, but satisfaction remains inherent in the use. Experiencing Symbolism in a variety of situations may enrich experiencing a built environment than perceiving instant static messages or naive ones. Social values that relate to moral and emotional values may create continuous interactive and variable social values that last.

\subsection{Truthfulness of meanings}


Although the client is usually the one paying fees to the architect, sincerity of the latter towards the users' benefit probably reaches higher goals. Therefore, intention in Islamic culture is a cornerstone of any work and promised to be rewarded even if the job failed. Although intentions are internal secrets, pretending a meaning can seldom escape declaration. Returning to fig. 1 , intentions of the gymnastic athlete either if cleverness is hiding self arrogance or aiming to raising her country's flag high, intentions will probably show themselves by the associational gestures and words sooner or later. The difference between patriotism and selfishness is large although the act is the same.. Media and publications have repeatedly presented the project at various stages during its completion. Jean Baudrillard pointed to manipulating minds as a tool of modernism in his book entitled "The gulf war did not take place", he argued in the book that "news events" introduce themselves as more significant than reality (Johnathan, 2000). In terms of positivism, media event can count as a valid component of reality, but forcing an idea through media event or publications will probably make credibility not last for long. Publicity and financial support to market an idea may help convince people's consciousness, but the unvarnished truth may easily find a way to be unconsciously credible. Therefore, the inexpensive project of the Memorial Wall, with the calmness of its site, and clarity of the idea constituted an easy open gateway to a spiritual acceptance.

\subsection{Experiencing architecture produces humanism}

Public buildings can depend on social values instead of short term pulsing emotional affect of symbolism. Familiarity may weaken passion of architectural symbols through daily use, but can be reversed into a place attachment when being a continuous locus of humanistic and social rapprochement. Architectural programming then plays an essential role in humanizing a place but usually does not add credit to the architect like sculpturing do. While essential activities are usually highlighted, associational activities may be more substantial to users. In the Faculty of Engineering building, students may spend more time in circulation spaces than in classes. Although the manifest behavior may seem to be the logic cause to be there, the latent behavior remains the real one. The architect's role is to choose which activities will prevail to take place and link an environment to values. Human, moral, and enjoyable values are preferable since are directly targeting feelings. For many students, friendship may add a delightful value in association to study activities and will strongly reflect to its place appreciation. Although the normal unnoticeable human body position in fig. 1 is miraculous in terms of balance, we may add that social comfort state of the couple's relaxed talk which has not drawn one's attention to the miracle.

\subsection{Conclusion}

Western societies tended to follow thoughts of modernism in all aspects of life including architecture which faithfully expressed it. The local Arab societies, among many others, appeared confused in following the vigorous materialism stream. The paper recalled humanities and rediscovered society potentials of authentic centripetal values, which used to characterize local traditions. 
Symbolism might be an approach to turn meanings into realistic buildings, and then one may retrieve the meanings every now and then, but when adjoined with function, symbolism would last for long. The authors argued that architecture should not be appreciated just as a piece of art. The paper invites architects to express values in a daily use context, and originally interact with people's feelings. By maintaining social ties, and addressing kindness, sincerity, and high morals, architecture may transcend beyond superficial or materialistic aesthetics. The task of an architect here becomes inclusive to the insight of the bonds and morals he/she can enhance and tailor through the design process, considering his/her deep attachment to those social patterns as if designing for oneself.

Aside symbolism, the authors consider particular local social interaction and human warmness a prospect to consist a base to human architecture as being a specific identity. The paper points to redefine architectural objectives according to a special broader understanding of material aesthetics towards a simple correlating social pleasure to built environments. When highlighting ethics to emphasize values, social mores, and social intimacy, the paper not only invites local architecture to liberate users' spirits in an appreciation independent of superficial formalism, but also persuades western architecture to reach similar benefits according to a positive cultural interaction basis.

Additional empirical research would explore preferences of humanistic spaces to widen scopes of design approaches in different cultures for different social groups in favor of humanity around the globe.

\section{References}

Adorno, Theodor (1998). Aesthetic theory. University of Minnesota Press, 35-118

Al- Masiri, Abdel Wahab, Al-Turayki, Fathy (2003). Modernism and post modernism (in Arabic). Dar Al fikr. Damascus, 189.

Al- Masiri, Abdel Wahab, (2011). Comprehensive secularism and partial secularism (in Arabic). Dar El Shorouk. Ed 4. Damascus, 69- 70.

Anonymous (2007). Public's Choice America's Best Architecture. AlArchitect. 14. February. 9.

Belk, Russell W. (1988). Third world consumer culture. In E. Kumcu \& A. F. Firat (Eds), Marketing and development: toward broader dimensions. Greenwich, CT. JAI Press, 103-127.

El Husseiny, Aly (1996). Children's image of school buildings. 1st International Conference, Faculty of Engineering, Alexandria University. Role of Engineering Towards Better Environment. RETBE 96.

El Husseiny, Aly (2004). Problematic of aesthetic preferences dissimilarity between the public and professionals, case Study: Historic Cairo region.(in Arabic) Engineering research journal. February.

Hale, Jonathan (2000). Building ideas, an introduction to architectural theory. John Wiley Sons, LTD. 1-2. Scribd. (n.d.). Retrieved from http://www.scribd.com/doc/62445834/Building-Ideas-Jonathan-Hale 
Hans-Peter Martin, Harald Schumann. (1996). Die globalisierungsfalle, der angriff auf demokratie und wohlstand" Rowohit Veerlag Gmbh, Germany. (translated into Arabic by Adnan abbas aly, Ramzi Zaki in Alam El Marefa 2003, 54-55.

Lang, Jon (1987). Creating architectural theory, the role of the behavioral sciences in environmental design. New York: Van Nostrand Reinhold.

Louise Marin, (1984) Utopics: spatial play, trans. by Robert A. Vollrath. Atlantic Highlands, NJ: Humanities Press. 239. 